\title{
Zur Frage der Sekundärinfektion tuberkulöser Nieren durch Eitererreger.
}

\author{
Von \\ Koji Yago. \\ (八于幸治) \\ (Aus der Chimurgischen Klinik von Prof. Sh. Sugimura \\ der Universität, Sendai.)
}

Das Vorkommen der Misch- bzw. Sekundïrinfektion bei tuberkulös crkrankter Niere durch pyogene Bakterien findet man in der Literatur vielfach erwähnt. Es scheint sich jedoch hierbei bisher meistens um Forschungen an Blasenharn oder seltener an katheterisierten Nierenharnen bei nierentuberkulösen Kranken zu handeln. Bakteriologische Untersuchungen direkt aus der operativ entfernten tuberkulösen Niere stammenden frischen Materials liegen nur in vereinzelten Fällen vor, so dass man hieraus betreffs der Misch- bzw. Sekundïrinfektion in der tuberkulösen Niere überhaupt kaum etwas erkennen kann.

Albarran machte schon im Jahre 1897 auf die Bedeutung der Sekundärinfution bei Uro-Tuberkulose aufmerksam. Er bemerkte, dass hierbei sehr hïufig Sekundärinfektion rorkommt und nicht nur die tuberkulös erkrankte Niere, sondern auch die zweite intakte Schwesterniere oft davon ergriffen ist. Solche Sekundirinfektion des Harns Nierentuberkulöser durch Eiterbakterien wurde nach Wildbol z in $22 \%$, nach Su te $r$ in 10,3\% (8 unter 78), nach Pousson in 37,5\% (12 unter 32), nach Runeberg in $13 \%$ und nach Rafin in $29,7 \%$ der Fälle beobachtet.

Unter seinen 10 Fïllen von Nierentuberkulose mit Mischinfektion konnte B. Frisch diese bei einem Fall direkt im Beckeninhalt einer operativ entfernten tuberkulösen Niere, bei den ubrigen überhaupt nur im ILarn der Kranken feststellen. Barney und Well es trafen bei 63 Nierentuberkulösen Selsundärinfektion in 50\% der Blasenharne und in $22 \%$ sämtlicher katheterisierter Nierenharne an. Dagegen konnte Spooner, der den Inhalt frisch entfernter tuberkulöser Nieren bakteriologisch untersuchte, keine Sekundürinfektion durch $\mathbf{E i -}$ tererreger nachweisen. Nur fand er bei Untersuchungen des Blasenharns 3 mal unter 7 Fälen neben Tuberkelbazillen noch andere Bakterien. An den direkt aus der erkrankten Niere stammenden Katheterhurnen konnte er keine Mischinfektion nachweisen.

Durchmustern wir die bisherige Literatur über dje Misch- bzw. Sekundärinfektion bei Nierentuberkulase und zwar der tuberkulös erkrank- 
ten Niere selbst, so treffen wir, wie oben erwähnt, auf wenige Angaben über bakteriologische Untersuchungen des Inhalts der exstirpierten tuberkulösen Niere selbst, die gerade für die Beurteilung solcher Infektion in tuberkulös erkrankter Niere wichtig wären. Auch über die Bezeichnung der mischinfizierten tuberkulösen Niere sind die Meinungen der Autoren geteilt. Wührend nach Koike Autoren wie Kapsammer, Zuckerkandl, Kusnetzky, Schlagintweit, Smirnow, Böhringer und Stoerk u. a. unter tuberkulöser Pyonephrose das Endstadium der käsig-kavernösen Tuberkulose, also eine Veründerung rein tuberkulösen Ursprungs verstehen, so lassen Autoren wie J. Israel, Wolff u. a. diese Bezeichnung nur für Fälle von Misch- bzw. Sekundürinfektion tuberkulös erkrankter Niere gelten. Wolff empfahl für die stark zerstörte, rein tuberkulöse Eiterniere die Bezeichnung ,sekundär pyogen infizierte, kïsig-kavernöse Nierentuberkulose," die der Nephropyelitis phthisica cum suppuratione nach $\mathrm{H}$. Ch iari entspricht.

Die Sekundärinfektion der einseitig tuberkulösen Niere steht der der zweiten intakten Niere an klinischer und vor allem prognostischer Bedeutung weitaus nach, da die erste gleichzeitig mit der operativen Entfernung der tuberkulösen Niere beseitigt werden kann. Aber dass die Sckundärinfektion der tuberkulösen Niere durch Eiterbakterien die wesentlichen Verlauf der Nierentuberkulose bösartig gestalten kann, wurde von A lbarran und einigen anderen Autoren hervorgehoben, während Autoren wie $W$ ildbolz u. a. dies auf Grund ihrer klinischen Erfahrungen bestreiten.

Da, wie aus Obigem hervorgeht, weitere Forschungen über diese Frage, auch vom klinischen Standpunkt aus, sehr wüuschenswert sind, so will ich auf Grund bakteriologischer Untersuchungen von 100 in unserer Klinik operativ entfernten tuberkulösen Nieren und der klinischen Beobachtungen dieser Fälle näher auf diese Frage eingehen und meine Resultate hier wiedergeben.

Bei meinen 100 Nierentuberkulosen wurde der Inhalt der Nierenkaverne oder des Nierenbeckens direkt nach der operativen Entfernung der tuberkulösen Niere unter ganz aseptischen Kautelen entnommen und bakteriologisch, d. h. teils tinkturell, teils kulturell untersucht. Diese bakteriologischen Untersuchungen wurden hauptsächlich im hiesigen bakteriologischen Institut vorgenommen, und ich fühle wich verpflichtet, auch hier dem Chef des Instituts, Herrn Prof. Dr. K. Aoki, meinen aufrichtigen Dank dafür auszusprechen. Alle diese exstirpierten tuberkulösen Nieren wurden ausserdem auch pathologisch-anatomisch und zwar histologisch untersucht, wodurch auch ihre tuberkulöse Natur bestitigt wurde. Ausserdem wurde bei Fïllen der Nierentuberkulose mit nachgewiesener Seliundärinfektion durch Eiterbakterien der Krankheitsverlauf ror und nach Nephrektomie genau verfolgt. 
Mein Material besteht aus 8 Fällen von Tuberkulose der Papillenspitze, 77 Fällen der käsig-kavernösen Form mit Einschluss ihres Endstadiums, d. h. der tuberkulösen Pyonephrose und der Kittniere, ferner aus 6 tuberkulösen Hydronephrosen, 8 Fällen der grobknotigen Form und einem Fall der fibrösen Form der Nierentuberkulose, so dass sie im ganzen 100 ausmachten. Darunter konnte ich, wie aus Tabelle I ersichtlich, in 13 Fällen, d. h. in 13\%, Misch- bzw. Sekundärinfektion der tuberkulösen Niere durch Fiterbakterien nachweisen (siehe Tabelle I).

\section{Tabelle I.}

II:ufigkeit der Sekundürinfektion bei verschiedenen anatomischen

Formen der Nierentuberkulose.

\begin{tabular}{|c|c|c|c|}
\hline Anatom. Formen & Positiv & Negativ & Summa \\
\hline $\begin{array}{l}\text { I. Tuberkulose der Papillenspitze } \\
\text { II. Käsig-kavernöse Forn \{lanfang } \\
\text { mittelschwer } \\
\text { Endstadium } \\
\text { III. Tuberkulöse Hydronephrose } \\
\text { IV. Grobknotige Form } \\
\text { V. Fibröse Form }\end{array}$ & $\begin{array}{l}3 \\
0 \\
5 \\
2 \\
2 \\
2 \\
1 \\
0\end{array}$ & $\begin{array}{l}5 \\
6 \\
40 \\
24 \\
4 \\
7 \\
1\end{array}$ & $\left.\begin{array}{c}8 \\
6 \\
45 \\
26\end{array}\right\} 77$ \\
\hline Total & 13 & 87 & 100 \\
\hline
\end{tabular}

Unter meinen 8 Tuberkulosen der Papillenspitze wurde die Mischinfektion bei 3 Fällen beobachtet (siehe Tabelle II). Dabei wurden bei Fall 1 Staphylococcus pyogenes aureus, bei Fall 2 Pyocyaneusbazillen fast in Reinkultur neben Tuberkelbazillen und bei Fall 3 Pyocyaneusbazillen kombiniert mit einer Coligruppe gefunden. Unter 45 mittelschweren, käsigkavernösen Nieren wurde die Mischinfektion bei 5 festgestellt, nämlich bei Fall 4 der Streptococcus viridans in Reinkultur, bei Fall 5 spärliche Streptokokken mit einer Pneumokokkengruppe, bei Fall 6 und 7 mehr oder minder zahlreiche Gram-positive Kokken und bei Fall 8 mässig zahlreiche Gram-negative Stäbchen, und zwar bei den letzten 3 Fällen nur im direkten Prïparat, nicht aber auf der Kultur und bei allen neben den Tuberkelbazillen. Unter 26 käsig-kavernösen Nierentuberkulosen in Endstadium war die Mischinfektion bei 2 festzustellen. Bei Fall 9 wurden nämlich Gram-negative Stäbchen und Gram-positive Kokken im Kittniereninhalt, bei diesen beiden nur im direkten Präparat gefunden, während die Kultur bei allen diesen negativ ausfiel. 
Ferner konstatierte ich unter 6 tuberkulösen Fydronephrosen nur bei 2 Mischinfektion, nämlich bei Fall 11 das Bacterium faecalis alkaligenes in Reinkultur und bei Fall 12 spärliche Proteusbazillen auf der Kultur des Hydronephroseinhalts. Unter 8 Füllen der grobknotigen Nierentuberkulose wurde die Misch- bzw. Sekundärinfektion nur bei einem Fall, d. h. bei Fall 13, und zwar zahlreiche Pyocyaneusbazillen neben spärlichen Tuberkelbazillen im Inhalt des wenig veräuderten Nierenbeckens gefunden.

Meine Untersuchungen zeigten, dass die Misch- bzw. Sekundürinfektion der tuberkulösen Niere durch Eiterbakterien weit häufiger bei Franen als bei Männern, d. h. bei 10 Frauen gegenüber 3 Mäunern, beobachtet wurde.

Was nun die mischinfizierenden Bakterienarten betrifft, so habe ich bei meinen Fällen als Eitererreger in 3 Fällen Pyocyaneusbazillen, danu Streptokokken, Streptococcus viridans, Staphylococcus pyogenes aureus, Pneumococeus, Proteusbazillen, Bacillus faecalis alkaligenes, Coligruppe, sonst auch Gram-positive Kokken und Gram-negative Stäbchen in vereinzelten Fiallen gefunden.

A lbarran fand bei der Sekundärinfektion tuberkulöser Nieren Colibazillen, Staphylo- und Streptokokken, lüufiger Colibazillen mit Staphylokokken vergesellschaftet, als Erreger. Unter seinen zwei als Beispiele angegebenen Fällen stellte er bei einer 17jährigen Patientin, deren Harn Colibazillen und Mikrokokken neben Tuberkelbazillen enthielt, Coli-Infektion der tuberkulösen Nierenkavernen fest, während bei einer 35jährigen Frau die Colibazillen und Staphylokokken neben den Tuberkelbazillen im Kaverneneiter der operativ entfernten tuberkulösen Niere nachgewiesen wurden. Bei diesem Fall fanden sich im Harn Colibazillen und Mikrokokken ausser den Tuberkelbazillen. Aus neuerer Zeit haben B. Frisch im Beckeninhalt einer exstirpierten tuberkulösen Niere, Gayet, Boeckcl und Frank, Iselin und Verliac im Eiter der abgeschlossenen tuberkulösen Niere Colibazillen als Sekundärinfektion bei je einem Fall festgestellt. Bei dem Fall von Boeckel und Frank wurde ein perinephritischer Abszess durch Staphylokokken zusammen mit der von Colibazillen mischinfizierten tuberkulösen Niere konstatiert. In meinen 13 Fïllen wurden im Inhalt des Nierenbeckens und der Kaverne der tuberkulösen Niere die oben angegebenen Bakterienarten angetroffen, von denen ich einige auch bei Nierensteininfektion gesehen babe. Ich konnte nämlich bei meinen 18 Fällen infizierter Steinniere oft Streptokokken, ausserdem auch vereinzelt Streptococcus viridans, Staphylococcus pyogenes aureus, Colibazillen, Gramnegative Stäbchen und Gram-positive Kokken nachweisen. 


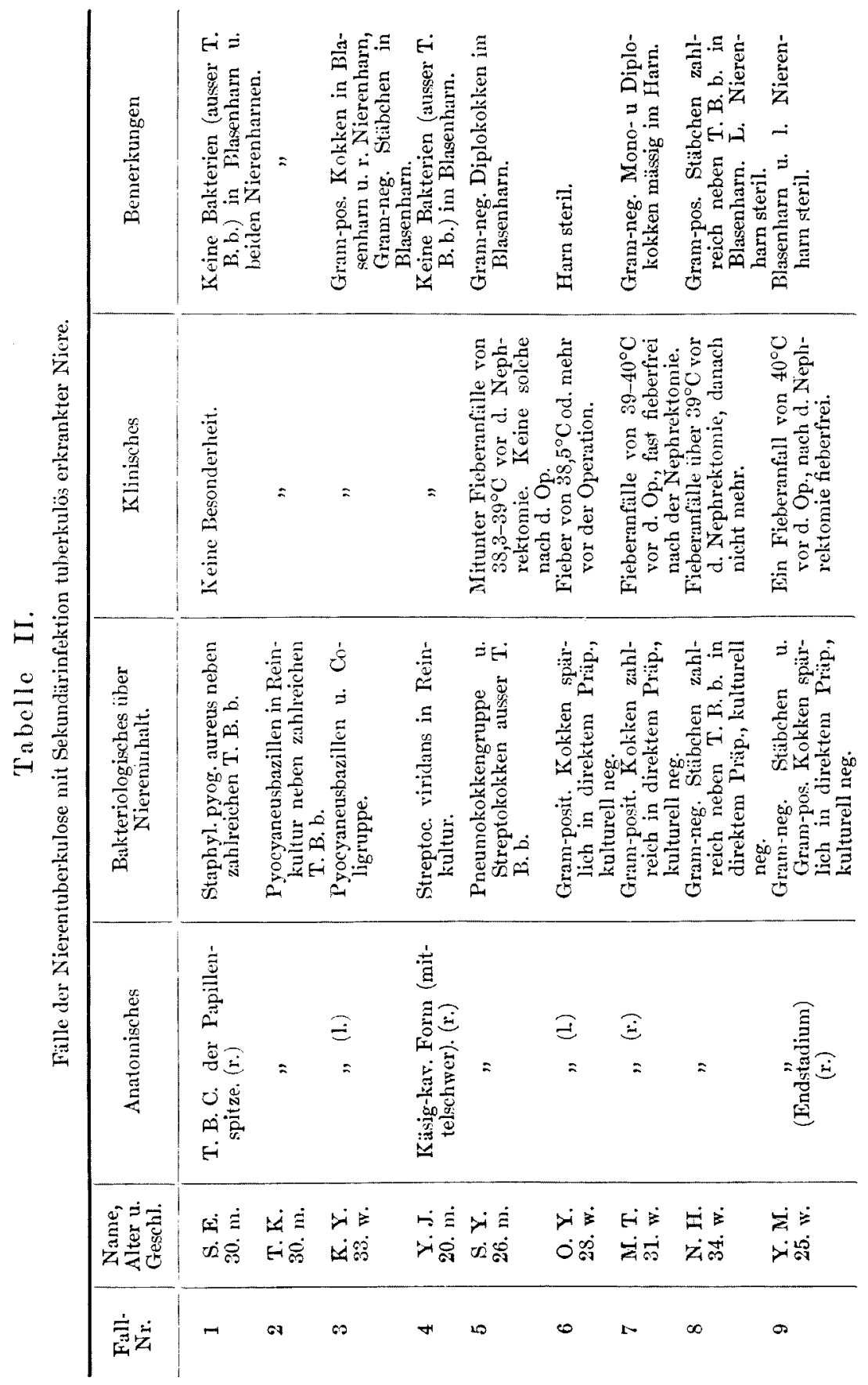



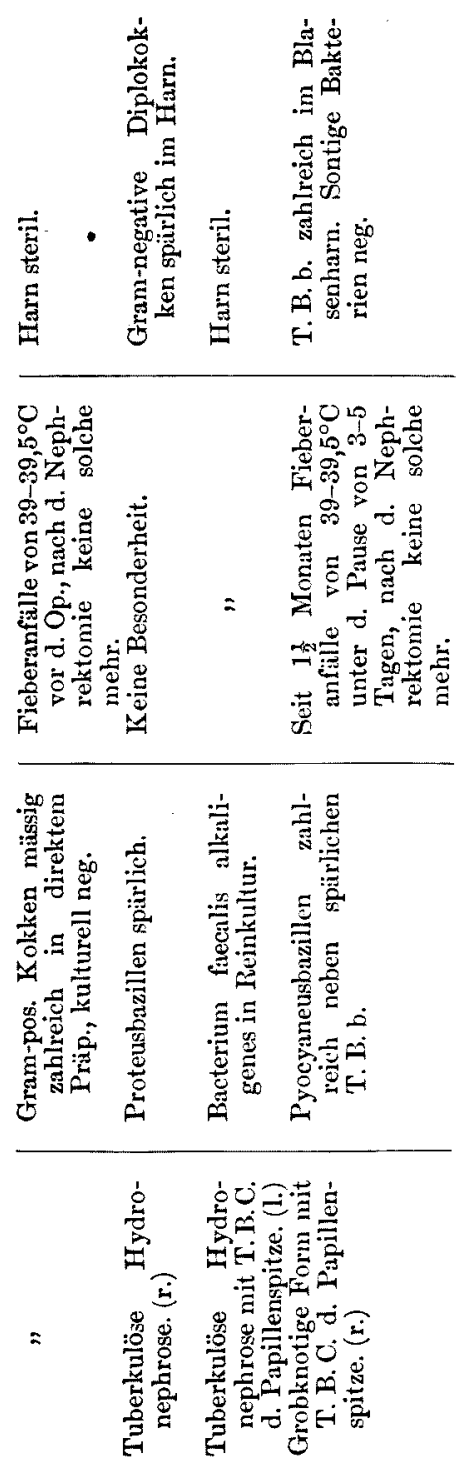

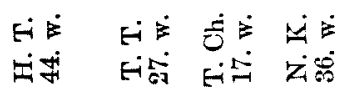

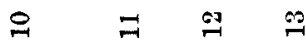

Hier möchte ich einige Worte über die anaerobe Infektion der tuberkulösen Niere hinzufiigen. Wildbolz beobachtete Fälle perirenalen Abszesses mit auffüllig stark stinkendem, grünem Eiter, der, auf dem üblichen Nährboden verimpft, kein Bakterienwachstum zeigte. Danach vermutet der Autor, dass öfters, als bis jetzt angenommen, eine Mischinfektion der tuberkulösen Niere mit anaeroben Bakterien erfolgt. In diesen von ihm erwähnten Fällen sollen die Patienten ror der Operation hohes Fieber, mit Temperaturen zwischen 39 u. $40^{\circ} \mathrm{C}$, gehabt haben und ihr Fieber nach Nephrektomie sofort gefallen sein. Runeberg bemerkte, dass bei Nierentuberkulose nicht selten anaerobe Bakterien im Harn nachgewiesen werden, und Young (nach David) gab verschiedene Anaerobien an, die überhaupt im Harn erscheinen können. In meinen Fällen konnte man kein besonderes Kulturverfahren für die Anaerobien anwenden. Howald hat neulich einen Fall von Sekundärinfektion tuberkulöser Niere durch den Frän kel schen Gasbrandbacillus berichtet.

Dass bei banaler Infektion der Harnwege verschiedene Eiterbakterien wie Colibazillen, Staphylound Streptokokken, Micrococcus tetragenus, Diplococcus pneumoniae, Enterococcus, Micrococcus catarrhalis, Proteus-, Pyocyaneus- und Influenzabazillen und auch Anaerobien vorkommen können, ist schon längst bekannt (Rovsing, Melchior, Baisch und Young). Im Urin Urotuberkulöser fand Rafin häufig Staphylokokken, seltener auch Colibazillen, Streptokokken, Pneumokokken und auch Tetragenus als Sekundärinfektion. Ähnliche Bakterien wurden hierbei auch von Pousson, B. Frisch und Runeberg u. a. festgestellt. In meinen 
Fällen (Fall 1, 2, 4, 6, 9, 10, 12 und 13) gab es, wie Tabelle II zeigt, oft im Blasenharn oder manchmal in separierten Nierenharnen keine mischinfizierten Eiterbakterien, obwohl sie im Inhalt der tuberkulösen Niere und des Nierenbeckens nachgewiesen wurden.

Auf welchem Wege nun diese Sekundärinfektion der tuberkulösen Niere erfolgte, das lässt sich bei meinen Fällen nicht leicht entscheiden. Diese Infektion kann, wie B. Frisch annimmt, sowohl auf exogenem (Katheterismus, Cystoskopie usw.) als auch auf endogenem Wege (Blutoder Lymphbahn) erfolgen. Dass exogene Harninfektion bei Urotuberkulose durch verschiedene Manipulationen begünstigt wird und diese leicht auch $\mathrm{zu}$ aszendierender Infektion der tuberkulösen Niere Veranlassung geben kann, wurde schon von Autoren wie Albarran, Legueu, Wildbolz u. a. erwähnt. Auch die Tatsache, dass bei meinen Fällen (3 Männer : 10 Frauen), wie bei Fällen von B. Frisch (2 Männer : 8 Frauen) die Frauen überwiegen, scheint dafür zu sprechen. Dass aber die Sekundärinfektion tuberkulöser Niere auch hämatogen entstehen kann, vermag ich auf Grund meiner oben angeführten Fälle nicht zu lengnen.

Albarran bemerkte, dass die Sekundärinfektion der tuberkulösen Niere auf zweierlei Weise, d.h. in anatomischer und klinischer Beziehung, den Verlauf der Nierentuberkulose beeinflussen kann. Dass hierbei anatomisch der Zerstörungsprozess des tuberkulös befallenen Nierenparenchyms infolge der eitrigen Sekundärinfektion schneller fortschreiten dürfte, nehmen auch Autoren wie Küster, Furniss, B. Frisch u.a. an, während Wildbolz keine regelmässige Erschwerung des Verlaufs als Folge davon beobachtete. Albarran, Legueu, Blumu. a. bemerkten bei solchen Fällen Verschlimmerung des wesentlichen Krankheitsverlaufs, d. h. hohes Fieber, zunehmende Kachexie und Schwäche. Auch paranephritische Abszessbildung wurde hierbei besonders von Blum, J. Israel, Runeberg u. a. hervorgehoben.

Bei meinen Fällen (Fall 5, 7, 8, 9 und 13) sind besonders hohe Fieberanfälle von $39-40^{\circ} \mathrm{C}$ und dementsprechende Störung des Allgemeinzustandes vor der Operation hervorzuheben, die aber bald nach erfolgter Nephrektomie völlig verschwanden. Ähnliche Fälle kann man auch unter den Fällen von B. Frisch finden (Fall 2 und 5). Klinisch lässt sich die erfolgte Sekundärinfektion in der tuberkulösen Niere vor der Operation manchmal sehr schwer feststellen, ja es ist beinah unmöglich, weil solch Fieberanstieg öfters bei Komplikationen von Lungen und Pleura, und zwar bei empfindlichen Personen hervorgerufen werden kann. Erst nach erfolgter Nephrektomie lassen sich manchmal leicht Rückschlüsse auf die 
wahre Sachlage ziehen, wie aus meinen oben angegebenen Fïllen ersichtlich.

\section{Zusammenfassung.}

1. Die Untersuchungen bisheriger Autoren über die Misch- bzw. Sekundärinfektion tuberkulöser Nieren durch Eiterbakterien betrafen meistens den Blasenharn oder seltener die separierten Nierenharme bei Nierentuberkulösen. Bisher liegen sehr wenige Angaben über bakteriologische Untersuchungen direkt aus der exstirpierten tuberkulösen Niere stammenden Materials vor.

2. Meine bakteriologischen Untersuchungen des Inhalts von tuberkulösen Niere und Nierenbecken betreffen 100 tuberkulöse Nieren, die in unserer Klinik operativ entfernt worden waren. Darunter fand ich in 13\% der Fälle Sekundärinfektion der tuberkulösen Niere durch Eiterbakterien.

3. Diese Sekundärinfektion wurde bei meinen Fällen in verschiedenen Formen bzw. Stadien der Nierentuberkulose und überwiegend bei Frauen (3 Männer : 10 Frauen) gefunden.

4. Als mischinfizierende Bakterien fand ich 3 mal Pyocyaneusbazillen, vereinzelt aber Streptokokken, Streptococcus viridans, Staphylococcus pyogenes aureus, Pneumococcus, Proteusbazillen, Bacillus faecalis alkaligenes, Coligruppe, sonst auch Gram-positive Kokken und Gram-negative Stäbchen.

5. Die Sekundärinfektion der tuberkulösen Niere könnte sowohl urogen als auch hämatogen erfolgen. Ihr Überwiegen bei Frauen in meinen Fällen macht es wahrscheinlich, dass die zuerst genannte Infektionsweise hierbei häufiger in Frage kommt.

6. Bei einigen meiner Fälle, d. h. bei 5 unter 13, lagen hohe Fieberanfälle von $39-40^{\circ} \mathrm{C}$ und dementsprechende Störung des Allgemeinzustands vor der Operation vor, die bald nach erfolgter Nephrektomie völlig verschwanden. Fieber infolge etwa vorhandener Komplikationen wie Lungen und Pleura war hierbei ausgeschlossen.

7. Es wäre aus theoretischen und praktischen Gründen zu empfehlen, solche sekundär infizierte tuberkulöse Niere unter der Bezeichnung „infizierte Nierentuberkulose" oder ,infizierte tuberkulöse Pyonephrose" von der zweideutigen Bezeichnung der tuberkulösen Pyonephrose zu unterscheiden, wie schon von früheren Autoren vorgeschlagen wurde. 


\section{Literatur.}

(1) Albarran, J.; Ann. d. malad. d. Org. gén.-urin., 1897, 1-18.

(2) Ba isch, K.; Beitr. z. Geburtsh. u. Gyn., 1904, 8, 297-328.

(3) Barne y, J. D. u. E. S. Welles; Journ. Amer. Med. Assoc., 1920, 74, 1499-1501.

(4) Blum, V.; Ztschr. f. Urol., 1909, 3, 264-265.

(5) Boeckel, A. u. A. Franck; Ref: Ztschr. f. urol. Chir., 1928, 25, 257.

(6) Frisch, B.; Ztschr. f. urol. Chir, 1929, 27, 248-256.

(7) Furniss, H. D.; Ref: Ztschr. f. urol. Chir., 1921, 8, 262.

(S) Gayet; Journ. d'urol., 1927, 23, 353-357.

(9) How ald, R.; Dtsch. Ztschr. f. Chir., 1930, 226, 152-155.

(10) Iselin u. Verli ac; Journ. d'urol., 1928, 25, 145-148.

(11) Israel, J. u. W. Israel; Chirurgie des Niere und der Harnleiters. Leipzig $1925,357-358$.

(12) Kodama, H. u. N. K rasnogorski; Ztrlbl. f. Bakt. Orig., 1913, 69, 8-22.

(13) Koike, M.; Mittl. u. allg. Path. u. path. Anat., 1927, 3, 540.

(14) K ïster, E.; Chirurgie der Nieren(D. Chir. Lief. 52 b), Stuttgart 1896-1902, 357.

(15) Legueu, F.; Traité chirurgicale d'urologie, 2. Aufl., Paris 1921, 829-830.

(16) Melchior, M.; Cystitis und Urininfektion, Berlin 1897, 78-79 u. 215.

(17) Pousson, A.; Précis des maladies des voies urinaires 4. Aufl, Paris 1917, $1074-1079$.

(18) Rafin ; Encyclopedie francaise d'urologie, 2, Paris 1814, 754.

(19) Rovsing, Th.; Die Blasenentzündungen, Berlin 1890, 35-43.

(20) Runeberg, B.; Ztschr. f. urol. Chir., 1927, 21, 282-283.

(21) Schwarzwald, R. Th. u. B. Frisch; Verhandl. d. Dtsch. Gesellsch. f. Urol. 5. Kongr., 1921, 92-93.

(22) Sugimura, Sh.; Tokyo Iji Shinshi, 1928, No. 2554, 10-12.

(23) Wildbol z, H.; Chirurgie der Nierentuberkulose (N. D. Chir. Bd. 6), Stuttgart $1913,63-69$.

(24) Wolff, A.; Ztschr. f. urol. Chir., 1921, 6, 332-335.

(25) Young, H. H.; Practice of Urology 1, Philadelphia u. London 1926, 90-109. 